\title{
Alocação Ótima de Geração Distribuída e Banco de Baterias para Restrição de Tensão e Redução de Perdas
}

\author{
Luíza S. Menezes*. Luan D. L. Pereira*. Lucas F. Encarnação*. Jussara F. Fardin*. Ícaro Henrique Honorato*. \\ Helder R. O. Rocha*. Rodrigo F**. \\ * Depto. de Engenharia Elétrica, Universidade Federal do Espírito Santo, Vitória, ES, Brasil (e-mail: \\ luiza.menezes@aluno.ufes.br,luamdiego@gmail.com, jussara.fardin@ufes.br, icaro.honorato@aluno.ufes.br, \\ helder.rocha@ufes.br. \\ ** Depto de Engenharia Elétrica. Instituto Federal do Espírito Santo, São Mateus, ES, Brasil (e- \\ mail:rodrigo.fiorotti@ifes.edu.br)
}

\begin{abstract}
This paperwork proposes a methodology to determine a model of distributed generation of electric energy from photovoltaic panels, determining the optimal allocation of these generators in an electric energy distribution network, using technical parameters and calculating the installation cost of those panels. The methodology makes use of the photovoltaic generation profile in the summer season and is based on the load curve of low voltage residential consumers. The optimization of the location of the distributed generation units aims to minimize the daily losses of active power and the installation costs of the generators connected to the network. The optimization problem also provides for the allocation of battery banks with the distributed generators, in order to manage the active power of the network and, consequently, to improve the voltage profile and minimize the electrical losses. The optimization solution is obtained through a Genetic Algorithm, which receives as input the daily data of the active power of the solar generators and the load demand to search for better solutions to the problem. To validate the proposed solution a test was carried out on a 78-bar radial feeder, resulting in daily losses of active power and voltage profiles.
\end{abstract}

\begin{abstract}
Resumo: Propõe-se uma metodologia para determinar um modelo de geração distribuída de energia elétrica a partir de painéis fotovoltaicos, determinando a alocação ótima desses geradores em uma rede de distribuição de energia elétrica, utilizando parâmetros técnicos e calculando o custo de instalação desses painéis. A metodologia faz uso do perfil de geração fotovoltaica na temporada de verão e é baseada na curva de carga de consumidores residenciais de baixa tensão. A otimização da localização das unidades de geração distribuída visa minimizar as perdas diárias de energia ativa e os custos de instalação dos geradores conectados à rede. O problema de otimização também prevê a alocação de bancos de baterias em concomitância com a geração distribuída, a fim de gerenciar a potência ativa da rede e, consequentemente, melhorar o perfil de tensão e minimizar as perdas elétricas. A solução de otimização é obtida através de um Algoritmo Genético, que recebe como entrada os dados diários da potência ativa dos geradores solares e a demanda de carga para buscar melhores soluções para o problema. Para validar a solução proposta, foi realizado um teste em um alimentador radial de 78 barras, resultando em perdas diárias de perfis de tensão e potência ativa.
\end{abstract}

Keywords: distribuited generation; distribuited generation allocation; genetic algorithm; battery allocation; distribuited systems.

Palavras-chaves: geração distribuída, alocação de geração distribuída, algoritmo genético, alocação de baterias; sistemas de distribuição.

\section{INTRODUÇÃO}

Distribuir energia elétrica nos tempos atuais é fundamental para que a sociedade possa manter o seu curso de desenvolvimento. Usa-se eletricidade para quase tudo: desde a agricultura, passando pelas indústrias de transformação, pelo comércio, residências e serviços públicos (ANEEL, 2016). Para tanto, no Brasil, existem as concessionárias e permissionárias de energia elétrica que, no que diz respeito à distribuição, são responsáveis por levar energia elétrica ao consumidor final.
No Brasil, as concessões e permissões de distribuição se enquadram no modelo econômico de mercado com monopólio regulado, ou seja, uma única empresa detém o comercio de distribuição de energia elétrica da região. Porém tal mercado é regulado pela Agência Nacional de Energia Elétrica (ANEEL) que, dentre suas finalidades, destaca-se a promoção do equilíbrio econômico-financeiro da concessão para a prestação de serviço, com qualidade adequada e preço justo (Fugimoto, 2010). 
Portanto, para que a energia distribuída seja de qualidade, a ANEEL determina que a tensão no ponto de entrega para o consumidor esteja entre 0.93 e 1.05 pu (ANEEL, 2010).

As distribuidoras, portanto, devem planejar a expansão da rede, bem como o investimento em equipamentos a serem instalados, de forma que as exigências do órgão regulador sejam atendidas. Além disso, sabendo que o retorno financeiro recebido pela distribuidora é de acordo com as perdas passíveis de gerenciamento, essa procura, sempre, diminuir tais perdas, de modo que haja um retorno maior (ANEEL, 2008).

Sendo assim, inúmeros estudos na área de redução de perdas da rede de distribuição são realizados, afim de atender às exigências do órgão regulamentador e aumentar $\mathrm{o}$ da concessionária lucro referente às perdas.

Nesse contexto, um artifício muito estudado é a alocação de baterias na rede, que promovem, além dos objetivos citados anteriormente, estabilidade na rede, melhor aproveitamento do sistema, aumenta a confiabilidade e possibilita à concessionária armazenamento de energia para um melhor controle (Oliveira, 2010). Tal contexto se justifica principalmente à época do verão, cuja incidência de radiação solar é mais elevada, ocasionando, não raro, a necessidade de armazenamento da energia excedente das GDs.

Devido aos custos associados aos sistemas de baterias observase que, na prática, a implementação de uma grande quantidade destes equipamentos espalhados pelos sistemas é inviável. Sendo assim, deve-se otimizar a alocação de baterias no sistema, bem como fazer o seu uso e controle de forma eficiente (Oliveira, 2010).

Um exemplo prático de utilização de algoritmo genético para melhor planejamento da rede de distribuição, é a alocação de Geração Distribuída na rede, visando, também, perda de energia e manutenção da tensão prevista pelo órgão regulador (Pereira, 2018).

\section{PROPÓSITO}

Desenvolver um método de busca de localização otimizada para a instalação das unidades de GD e banco de baterias na rede de distribuição, com a finalidade de minimizar as perdas diárias de potência ativa, o custo global do investimento e garantir que as baterias mantenham níveis de carregamento mínimos para maior vida útil.

\section{MÉTODOS}

Nessa secção expõe-se a metodologia utilizada para determinar a alocação ótima das unidades de GD e dos bancos de baterias em alimentadores radiais, objetivando minimizar os custos globais.

As principais etapas para realizar a metodologia proposta são: determinar a potência das unidades de GD; definir o perfil de carga adotado para as simulações e determinar a localização ótima das unidades de GD e dos bancos de bateria no alimentador de 78 barras com base em um algoritmo genético (AG).

\subsection{Definição da Potência das Unidades Solares}

A potência gerada nos painéis solares depende da temperatura do local, da irradiação solar, e das características físicas do módulo fotovoltaico. Para mensurá-la foi utilizada a metodologia a seguir (Atwa et al., 2010) cujas equações matemáticas são descritas em (1) - (6).

$$
\begin{aligned}
& T_{c}=T_{a}+r\left(\frac{N_{o t}-20}{0,8}\right) \\
& I=r\left[I_{s c}+K_{i}\left(T_{c}-25\right)\right] \\
& V=V_{o c}-K_{v} \times T_{c} \\
& F F=\frac{V_{m p p} \times I_{m p p}}{V_{o c} \times I_{s c}} \\
& P_{S}=F F \times V \times I
\end{aligned}
$$

Sabendo que $T_{c}$ é a temperatura do painel fotovoltaico $\left({ }^{\circ} \mathrm{C}\right)$, ), $\mathrm{V}$ a tensão no módulo fotovoltaico $(\mathrm{V}), V_{o c}$ a tensão de circuito aberto do módulo $(\mathrm{V}), N_{o t}$ a temperatura nominal de operação da célula $\left({ }^{\circ} \mathrm{C}\right), K_{i}$ o coeficiente corrente/temperatura $\left(\mathrm{A} /{ }^{\circ} \mathrm{C}\right)$, $T_{a}$ a média da temperatura ambiente no período $\left({ }^{\circ} \mathrm{C}\right)$, I a corrente na célula (A), $I_{s c}$ a corrente de curto-circuito da célula (A), $K_{v}$ o coeficiente tensão/temperatura $\left(\mathrm{V} /{ }^{\circ} \mathrm{C}\right), \mathrm{FF}$ o Fator de forma (adimensional), $V_{m p p}$ a tensão no ponto de máxima potência (V), $I_{m p p}$ a corrente no ponto de máxima potência (A) e $P_{S}$ a potência gerada pelo painel $(\mathrm{W})$.

Sendo assim, com base em (Oliveira, 2002) e em (Dos Santos et al., 2008), calculou-se a potência em pu, por hora, gerada por cada unidade de GD, obtendo-se um vetor de 24 posições, representando a geração solar de um dia.

\subsection{Definição da Curva de Demanda}

O perfil de carga do alimentador foi definido de acordo com o exposto em (Dos Santos et al., 2008), de modo que o pico de consumo seja no horário de ponta, 18 horas às 21 horas (PRORET, 2011) e o pico de geração seja das 12 às 14 horas.

\subsection{O Alimentador e o Fluxo de Carga}

Para a obtenção do fluxo de potência, ou fluxo de carga, representou-se a rede de maneira radial e fez-se uso de um alimentador de 78 barras (Rocha et al., 2009). Este é representado por um conjunto de inequações e equações, cujo fluxo é calculado a cada hora do dia levando em consideração a geração horária das GDs e o armazenamento de carga dos bancos de bateria.

Para tanto, foi utilizado o método da soma de potência, adaptado de (Broadwater, 1988). Esta metodologia faz uso de um processo de solução que possui um ótimo desempenho para resolver problemas de fluxo de carga em alimentadores radiais.

O algoritmo para obter o fluxo é tal que são definidos os parâmetros do sistema, como a identificação da tensão da subestação (nó de referência) e a tolerância para convergência $(\varepsilon)$. Em seguida definem-se os valores das tensões dos nós igual à tensão de referência, considerando as perdas totais nulas e calcula-se a soma das potências em cada nó (iniciando pelos nós mais extremos e percorrendo até a subestação), incluindo as perdas obtidas em (6) e (7); onde $P_{k m p}$ e $Q_{k m p}$ 
são as perdas ativas e reativas entre as barras k e m, $r_{k m}$ e $x_{k m}$ são a resistência e reatância do trecho $\mathrm{km}, P$ e $Q$ são as potências ativa e reativa da carga e $V_{m}$ é o módulo da tensão na carga.

Em seguida calcula-se os novos valores de tensão em todas as barras do sistema, agora iniciando pela subestação e percorrendo até os nós periféricos, por meio de (8); onde $V_{m} \mathrm{e}$ $V_{k}$ são as tensões dos nós k e m, $r_{k m}$ e $x_{k m}$ são a resistência e reatância do trecho km e $P$ e $Q$ são as potências ativa e reativa das cargas, para se calcular os novos valores de perda.

O critério de convergência é tal que se a diferença entre as perdas totais da iteração atual e da iteração anterior for menor que o valor de tolerância para convergência, finda-se o processo. Caso tal condição não ocorra, calcula-se a soma das potências de cada nó novamente e o processo é repetido.

$P_{k m p}=r_{k m} \frac{\left(P^{2}+Q^{2}\right)}{V_{m}^{2}}$

$Q_{k m p}=x_{k m} \frac{\left(P^{2}+Q^{2}\right)}{V_{m}^{2}}$

$V_{m}^{4}\left[2\left(r_{k m} P+x_{k m} Q\right)-V_{k}^{2}\right] V_{m}^{2}+\left(P^{2}+Q^{2}\right)\left(r_{k m}^{2}+x_{k m}^{2}\right)=$ 0

\subsection{Alocação Ótima}

Neste trabalho optou-se pela possibilidade de alocação separada dos Bancos de Bateras e de GDs nas barras, desde que a alocação destes retornassem as menores perdas. A presença de Bancos de Baterias na rede elétrica permite manter a oscilação da potência ativa na rede, provocada pelo perfil de carga durante o dia, em níveis menores, provocando, no alimentador, níveis de tensão mais próximos nas diferentes horas do dia e mantendo um nível de tensão mais linear entre as barras.

A população inicial é gerada de maneira randômica pelo algoritmo genético, visto que os indivíduos dessa serão utilizados para a geração da próxima população. O processo é tal que cada dois cromossomos são pais de outros cromossomos.

Esses novos cromossomos surgem por meio de Crossovers entre seus pais e Mutação Genética feitas logo após as operações de Crossover. Esses novos indivíduos farão parte de uma nova população e serão associados, cada um, a um custo.

Em seguida, a nova população de indivíduos será reduzida para o tamanho da população inicial, de modo que só os indivíduos com os menores custos permaneçam

Como entrada do AG, têm-se um vetor com 24 valores de potência ativa oriundos das unidades de GD e um vetor com 24 valores de potência ativa oriundos do perfil de carga adotado. A localização das unidades de GD é representada por um cromossomo com $x$ bits, sendo $\mathrm{x}$ a quantidade de barras do alimentador e a localização dos bancos de bateria também é representada por um cromossomo de x bits, de maneira que estes sejam alocados após a escolha da alocação da unidade de GD. A codificação utilizada é a binária, onde 1 indica a presença de GD e Banco de Bateria.
Ao inserir uma unidade de GD em uma determinada barra k, será possível suprir a carga desta barra e fornecer o excedente para o sistema, quando necessário. O mesmo ocorre com a inserção do banco de bateria: caso a potência existente na barra seja capaz suprir a carga da barra, a bateria será carregada; caso contrário, a bateria irá injetar energia na barra. As modificações necessárias para representar a presença da GD e do Banco de Baterias são mostradas em (9) e (10), respectivamente, onde $\Delta P_{k}$ é a variação de potência ativa (W) resultante na barra $\mathrm{k}, P_{D k}$ é a potência ativa (W) demandada na barra k, $G D_{k}$ é a variável binária para determinar a presença de GD na barra k. O mesmo ocorre no caso da inserção de um banco de baterias, com a exceção de que o valor injetado na rede, ou armazenado da rede, é determinado pelo percentual de carregamento da bateria, bem como pelos limites de tensão na barra. O fluxograma do funcionamento da bateria encontrase na figura 1 .

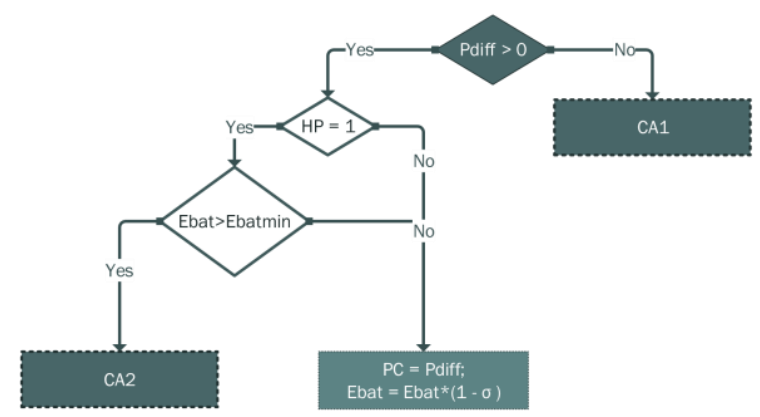

Figura 1. Fluxograma de Funcionamento da Bateria.

Onde CA1 e CA2 são dois conjuntos de ações que significam, respectivamente, o conjunto de ações tomadas quando a geração de energia renovável é maior que a demanda de energia e o conjunto de ações tomadas quando a demanda é maior que a geração, o horário é de ponta e a energia presente na bateria é maior que a energia mínima, o que permite sua utilização. Estes dois conjuntos são apresentados a seguir, nas Figuras 2 e 3.

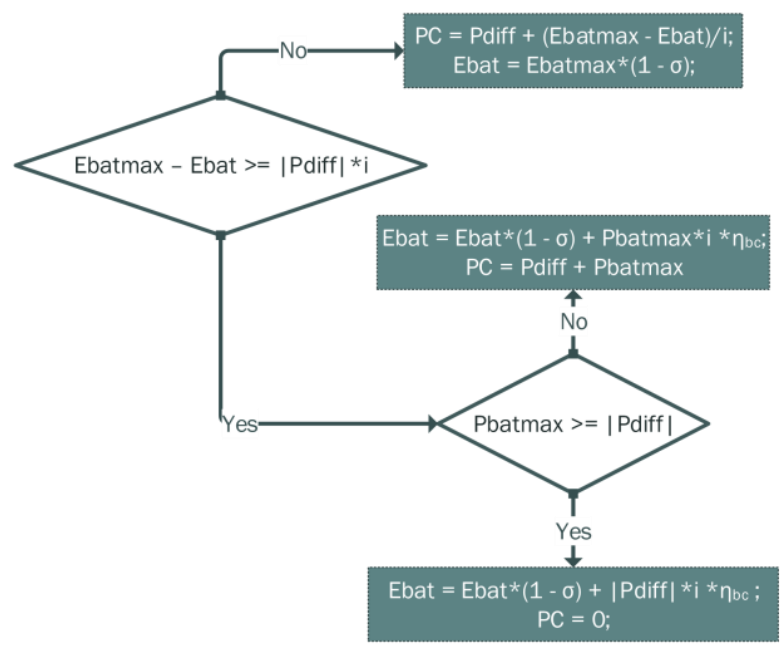

Figura 2. CA1 


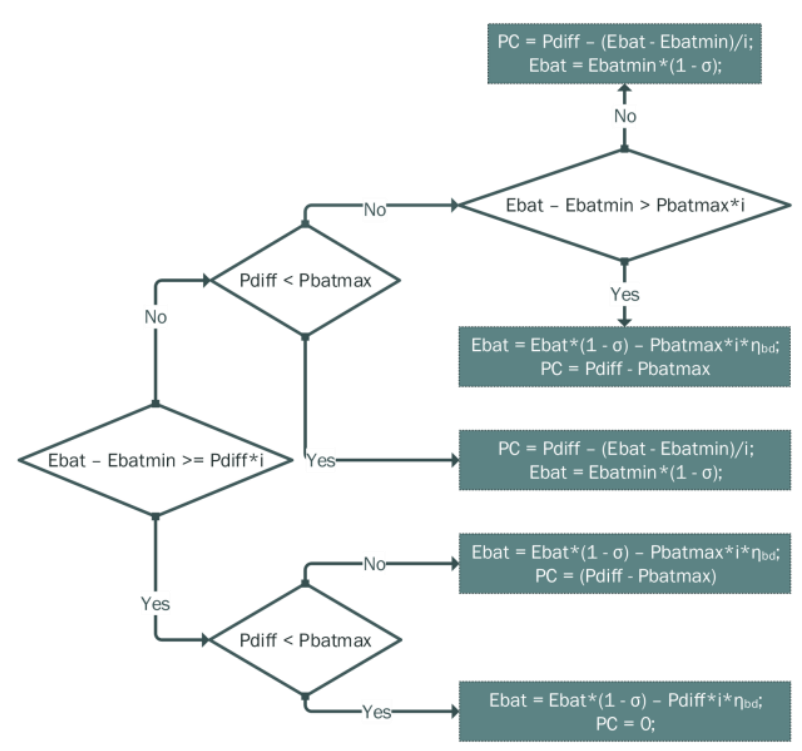

Figura 3. CA2

A determinação do armazenamento de energia na bateria ou da descarga promovida por essa na barra em questão tem as seguintes premissas:

(1) Analisa-se se o horário em questão é um horário de ponta ou fora de ponta;

(2) Caso seja um horário fora de ponta, opta-se por armazenar energia na bateria, desde que os níveis de tensão das barras sejam mantidos dentro do regulatório;

(3) Caso seja um horário de ponta e a demanda ultrapasse a geração, verifica se a bateria tem carga suficiente para injetar na rede e não danificar a vida útil da mesma.

$\Delta P_{k}=P_{D k}-G D_{k} \times P_{G D k}$

$\Delta P_{k}=P_{D k}-B T_{k} \times P_{B T k}$

O mesmo se repete para as 78 barras 24 vezes.

\subsection{Formulação Matemática do Problema}

Para melhor alcance do objetivo, otimização da alocação das GDs, dos Bancos de Bateria e do gerenciamento da carga da bateria, além de minimizar as perdas de potência ativa, é preciso também minimizar o custo global do projeto. Para tanto, optou-se por uma formulação mono-objetivo que integra as perdas de potência ativa e o custo global em uma única função.

Na proposta, busca-se minimizar as perdas anuais de potência ativa dos alimentadores, assim como o custo global de implantação do projeto. São considerados os custos relacionados à instalação e manutenção das unidades de GD e dos Bancos de Bateria, além de respeitar os limites máximos de tensão nas barras conforme estipulado pelo PRODIST.
Para cada intervalo horário a inserção de GD e Banco de Bateria deve atender à restrição do balanço de potência ativa e reativa nas barras do alimentador, conforme (11) e (12), respectivamente, sempre levando em consideração a carga ou descarga da bateria conforme o horário analisado.

$$
\begin{gathered}
\Delta P_{k}=G D_{k} \times P_{G D}-P_{D k}-P_{k}(V, \theta)=0 \\
\Delta P_{k}=B T_{k} \times P_{B T}-P_{D k}-P_{k}(V, \theta)=0
\end{gathered}
$$

$\mathrm{O}$ número de unidades de GD e de Bancos de Baterias instalados no alimentador é limitado pelas restrições mostradas em (13) e (14), onde $G D_{k}$ é a variável binária para determinar a presença de GD na barra $\mathrm{k}, B T_{k}$ é a variável binária para determinar a presença de Bancos de Baterias na barra k, $N_{G D}$ é o número máximo permitido de GD no sistema e $N_{B T}$ é o número máximo permitido de Bancos de Baterias no sistema.

$$
\begin{aligned}
& \sum_{k=2}^{n b} G D_{k}=N_{G D} \\
& \sum_{k=2}^{n b} B T_{k} \leq N_{B T}
\end{aligned}
$$

\subsection{Função Objetivo}

O AG proposto utiliza como função objetivo (FO) minimizar o custo global com a instalação das unidades de GD, dos Bancos de Bateria e perdas diárias de potência ativa do sistema e atender às restrições de operação da rede, de acordo com (15).

$$
\begin{aligned}
& F O=\min \left(\left(\alpha_{1} C_{\text {global }}-\alpha_{2}\left(\sum_{i=1}^{96} \Delta P_{\text {perdas }}^{i}+P_{G D}^{i}\right) \times t_{e}\right)+\right. \\
& \left.\beta \sum_{i=1}^{96}\left(\sum_{i=1}^{n b}\left|\Delta V^{k}\right|\right)_{i}\right)
\end{aligned}
$$

Onde $\Delta P_{\text {perdas }}^{i}$ representa a redução das perdas de potência ativa no intervalo horário $\mathrm{i}$ em relação às perdas originais do alimentador antes da inserção de GD e Bancos de Baterias, $P_{G D}^{i}$ representa a potência ativa gerada pelas unidades de GD no intervalo horário i, $t_{e}$ é a tarifa da energia comprada pela concessionária, $C_{\text {global }}$ representa o custo de instalação e manutenção das unidades de GD e dos Bancos de Baterias e $\Delta V^{k}$ representa os desvios de tensão no k-ésimo nó da rede. As constantes $\alpha_{1}, \alpha_{2}$ e $\beta$ são as penalidades impostas aos termos da função objetivo de forma a obter a melhor solução para o problema de otimização.

\section{Tabela 1. Valores das Constantes da Função Objetivo}

\begin{tabular}{cc}
\hline Constante & Valor \\
\hline$\alpha_{1}$ & 1 \\
$\alpha_{2}$ & 2 \\
$\beta$ & 4 \\
$t_{e}$ & 0,56228 \\
$C_{\text {global }}$ & $\mathrm{R} \$ 40.000,00$ \\
\hline
\end{tabular}

\section{RESULTADOS}

Nessa secção serão apresentados os resultados obtidos pelo algoritmo genético a cada vez que ele foi rodado. Para 
constatação dos resultados, o algoritmo foi rodado 10 vezes para cada caso e percebeu-se que as convergências aconteciam sempre próximas dos resultados aqui observados. Assim sendo, o número de máximo de iterações, bem como a população escolhida, foi encontrado por meio de testes, até que as convergências acontecessem, por 10 vezes seguidas, nas mesmas condições de custo e iterações. Os valores de base adotados para potência e tensão são de 1 MVA e 13,8 kV, respectivamente.

\subsection{Caso Base: Nenhuma Unidade de GD e nenhuma bateria}

Neste cenário considera-se que nenhuma unidade de GD está conectada ao sistema e o valor das perdas anuais de potência ativa para os alimentadores é de, aproximadamente, 44,70 p.u. para um sistema de 78 barras.

Observou-se que os níveis de tensão na maioria das barras do alimentador, para ambos os alimentadores, são críticos, afetando diretamente a qualidade de energia entregue ao consumidor, além de gerar multa para a concessionária por violação de tensão, conforme a figura 4 . Na figura 4 também observam-se os perfisde tensão observados na inserção de GDs e bancos de baterias, confirmando que a alocação de 3 GDs e 3 baterias proporcionam um melhor perfil de tensão.

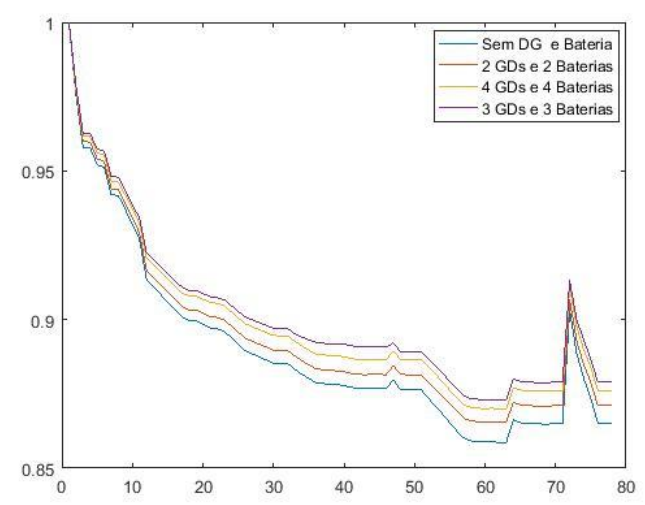

Figura 4. Perfis de tensão

\subsection{Alocação de uma bateria}

A alocação de uma bateria proporcionou os resultados conforme a tabela 2. Observa-se, ainda, que o algoritmo genético adotado não é o mais adequado para otimizar a alocação de uma bateria, visto que o custo, com o passar das iterações, permanece o mesmo e o algoritmo não converge.

\subsection{Alocação de duas baterias}

A alocação de duas baterias proporcionou os resultados conforme a tabela 2. Observa-se, ainda, que o algoritmo genético possui convergência em torno de 10 iterações e que o melhor resultado de função objetivo encontrado está entre 13,8 e 13,9 .

\subsection{Alocação de três baterias}

A alocação de três baterias proporcionou os resultados conforme a tabela 2. Observa-se, ainda, que algoritmo genético possui convergência em torno de 20 iterações e que o melhor resultado de função objetivo encontrado está entre 13,7 e 13,8.

\subsection{Alocação de quatro baterias}

A alocação de quatro baterias proporcionou os resultados conforme a tabela 2. Observa-se, ainda, que o algoritmo genético possui convergência em torno de 10 iterações e que o melhor resultado de função objetivo encontrado está em torno de 14 .

Tabela 2. Alocação das Baterias

\begin{tabular}{cccc}
\hline Número de Baterias & $\mathbf{2}$ & $\mathbf{3}$ & $\mathbf{4}$ \\
\hline Redução de & 10,97 & 10,95 & 10,96 \\
$\begin{array}{c}\text { Perdas (kW) } \\
\text { Redução }\end{array}$ & 24,56 & 24,52 & 24,54 \\
percentual (\%) & & $9 ; 10 ; 1$ & $8 ; 10 ; 11 ;$ \\
Barras com Baterias e & $8 ; 12$ & 1 & 12 \\
\hline GDs & & &
\end{tabular}

\section{CONCLUSÃO}

Neste trabalho foi apresentada uma metodologia para obter a localização ótima das unidades de GD através do método meta-heurístico AG. O objetivo da otimização foi minimizar as perdas anuais de potência ativa e o custo global de investimento, considerando as restrições características do problema.

Os resultados mostraram que a metodologia proposta para otimizar a alocação das unidades de GD, em conjunto com os Bancos de Bateria, reduz significativamente as perdas anuais de potência ativa do sistema. Dentre os diferentes cenários de testes, àqueles com o maior número de unidades de GD obtiveram menores perdas e um maior retorno financeiro, atendendo às restrições técnicas e operacionais.

A metodologia de previsão de carga e de otimização da alocação das unidades de GD constitui uma importante ferramenta para o planejamento do SEP.

\section{AGRADECIMENTOS}

Os autores agradecem à CNPQ, FAPES e à CAPES pelo suporte financeiro.

\section{REFERÊNCIAS BIBLIOGRÁFICAS}

\section{AGÊNCIA NACIONAL DE ENERGIA ELÉTRICA.} Submódulo 7.1 - PRORET, 2011.

Atwa, Y.M.; El-Saadany, E.F.; Salama, M.M.A.; Seethapathy, R., "Optimal renewable resources mix for distribution system energy loss minimization," IEEE Trans. Power Syster 1 (2010) 360-370.

Azevedo, G.A., "Geração Distribuída: Uso da Energia Solar em Condomínios de Edifícios," ISSN 2179-5568 - 
Revista Especialize On-line IPOG - Goiânia - Edição nº 11 Vol./2016 julho//2016.

Brasil, "Resolução Normativa $\mathrm{N}^{\circ} 338$, de 25 de Novembro de 2008" Agência Nacional de Energia Elétrica - ANEEL: Brasília, 2008.

Brasil, "Procedimentos de Distribuição de Energia Elétrica do Setor Elétrico" Agência Nacional de Energia Elétrica ANEEL: Brasília, 2010.

Brasil, "A Tarifa de Energia Elétrica" Agência Nacional de Energia Elétrica - ANEEL: Brasília, 2016.

Brasil, "Procedimentos de Distribuição de Energia Elétrica no Sistema Elétrico Nacional - Módulo 1: Introdução," de 01 de Março de 2016. ANEEL. Brasília.

Broadwater, P.R. et al., "Power Flow Analysis of Unbalaced Multiphase Radial Distribution Systems", Electric Power Systems Research Journal, Vol. 14, 1988.

Direito, L. C. M., “Alocação ótima de bancos de capacitores em redes de distribuição de energia elétrica," Dissertação (mestrado) Universidade Federal Fluminense, 104 f., 2010.

Dos Santos, I. P.; Junior, J. U.; Rüther. Energia Solar Fotovoltaica Como Fonte Na Busca Da Sustentabilidade, 2008.

L.D.L. Pereira, “Alocação Ótima de Geração Distribuída em Sistemas de Distribuição Considerando Incertezas no Modelo probabilístico de Geração", Programa de PósGraduação em Engenharia Elétrica, UFES, 2018.

Monticelli, A. J. "Fluxo de Carga em Redes de Energia Elétrica," Editora E. Blucher, Centro de Pesquisas de Energia Elétrica, Rio de Janeiro, 1983.

OLIVEIRA, S. H. F. DE. Geração Distribuída De letricidade; Inserção De Edificações Fotovoltaicas Conectadas À Rede No Estado De São Paulo, 2002.

Rocha, H. R. O.; Augusto, A. A.; Direito, L. C.; Souza, J. C. S.; Coutto Filho, M. B. "Alocação De Capacitores Em Redes Elétricas Via Heurística Construtiva E Refinamento Por Busca Tabu," XLI Simpósio Brasileiro de Pesquisa Operacional, Porto Seguro, 2009.

S. K. Fugimoto, "Estrutura de tarifas de energia elétrica: análise crítica e proposições metodológicas," Escola Politécnica da Universidade de São Paulo, São Paulo, 2010

T.T Oliveira, D.R.R. Penido, L.R Araújo, “Optimal Allocation and Battery Operations for Voltage Constraint and Loss Reduction" IEEE Latin American Transactions, IEEE, vol. 16, No ${ }^{\circ}$ 7, July 2008, 2010.

Thapar, V.; Agnihotri, G.; Sethi, V. K. "Critical analysis of methods for mathematical modelling of wind turbines," Renewable Energy, Vol. 36, No. 11, pp. 3166- 3177, 2011. 